\title{
BMJ Open Adherence to antibiotic guidelines and reported penicillin allergy: pooled cohort data on prescribing and allergy documentation from two English National Health Service (NHS) trusts
}

Cameron J Phillips, ${ }^{01,2,3,4,5}$ Mark Gilchrist, ${ }^{1,2}$ Fiona J Cooke, ${ }^{3}$ Bryony D Franklin, ${ }^{2,6,7}$ David A Enoch, ${ }^{3,4}$ Michael E Murphy, ${ }^{3}$ Reem Santos, ${ }^{8}$ Eimear T Brannigan, ${ }^{1}$ Alison $\mathrm{H} \mathrm{Holmes}^{1,2}$

To cite: Phillips CJ, Gilchrist M, Cooke FJ, et al. Adherence to antibiotic guidelines and reported penicillin allergy: pooled cohort data on prescribing and allergy documentation from two English National Health Service (NHS) trusts. BMJ Open 2019;9:e026624. doi:10.1136/ bmjopen-2018-026624

- Prepublication history and additional material for this paper are available online. To view these files, please visit the journal online (http://dx.doi org/10.1136/bmjopen-2018026624).

Received 12 September 2018 Revised 14 December 2018 Accepted 8 January 2019

Check for updates

(C) Author(s) (or their employer(s)) 2019. Re-use permitted under CC BY-NC. No commercial re-use. See rights and permissions. Published by BMJ.

For numbered affiliations see end of article.

Correspondence to Cameron J Phillips; Cameron.Phillips@flinders. edu.au;

Cameron.Phillips@imperial. ac.uk

\section{ABSTRACT}

Objective To investigate documentation of antimicrobial allergy and to determine prescribing adherence to local antibiotic guidelines for inpatients with and without reported penicillin allergy treated for infection in a National Health Service (NHS) context.

Setting Data were collected at two English hospital NHS trusts over two time-periods: June 2016 and February 2017.

Design Cohort study. Trust 1 data were sourced from prospective point prevalence surveys. Trust 2 data were extracted retrospectively from an electronic report. Participants Inpatients treated for urinary tract infection (UTI), community-acquired pneumonia (CAP), hospital-acquired pneumonia (HAP) and skin and soft tissue infection (SSTI). Data on allergy were collected, and antibiotic selection assessed for adherence to trust guidelines with differences between groups presented as adjusted ORs.

Results A total of 1497 patients were included, with 2645 antibiotics orders. Patients were treated for CAP $(n=495$; $33.1 \%)$, UTI (407; 27.2\%), HAP (330; 22\%) and SSTI (265; $17.7 \%)$. There were $240(16 \%)$ patients with penicillin allergy. Penicillin allergy was recorded as allergy $(n=52$; $21.7 \%)$, side effect $(27 ; 11.3 \%)$ and no documentation (161; 67.1\%). Overall, 2184 (82.6\%) antibiotic orders were guideline-adherent. Adherence was greatest for those labelled penicillin allergy (453 of $517 ; 87.6 \%$ ) versus no allergy (1731 of $2128 ; 81.3 \%$ ) (OR 0.52 (95\% Cl 0.37 to $0.73) p<0.001)$. Guideline-adherence for CAP was higher if penicillin allergy (151 of 163; 92.6\%) versus no allergy (582 of $810 ; 71.9 \%$ ) (OR 0.20 (95\% Cl 0.10 to 0.37 ) p<0.001). There was no difference in adherence between those with and without penicillin allergy for UTI, HAP or SSTI treatment. Conclusions A relatively high proportion of patients had a penicillin allergy and two thirds of these had no description of their allergy, which has important implications for patient safety. Patients with penicillin allergy treated for $\mathrm{CAP}$, received more guideline adherent antibiotics than those without allergy. Future studies investigating the clinical impact of penicillin allergy should include data on adherence to antibiotic guidelines.
Strengths and limitations of this study

- This study contains multisite data providing adherence rates to antibiotic guidelines for inpatients with penicillin allergy treated for active infection.

- This cohort study provides data on the extent of penicillin allergy documentation in a large National Health Service cohort which is meaningful as much of the data on this topic is from outside England.

- The infections captured in this study account for half of all indications requiring antibiotic treatment in hospital, which gives strength to generalisability to our findings.

- Confounding bias cannot be excluded as no information was collected on comorbidities, presence of febrile illness or immune suppression which may have influenced selection of antibiotics.

\section{INTRODUCTION}

Penicillin allergy can be a serious and life threatening reaction and reduces the choice of antibiotics available to treat infection with this first-line antibiotic. ${ }^{1}$ It is reported that $10 \%$ of the UK population have a penicillin allergy with approximately one-fifth of these being a true allergy, ${ }^{2}$ and many deemed spurious. ${ }^{4}$ Penicillin allergy is frequently documented in hospitalised patients, ${ }^{56}$ yet few patients with a reported penicillin allergy ever get this diagnosis verified. ${ }^{7}$ The importance of clinicians elucidating a clear history (of specific agent, dose, duration, onset and resolution of symptoms) have been well stated. ${ }^{28}$ Unfortunately, there is inconsistency in the documentation of antimicrobial allergies by practitioners and the details of allergy are frequently not recorded. ${ }^{9}$

Studies have reported that patients with a reported penicillin allergy have extended 
length of hospital stay, poorer clinical outcomes, increased risk of secondary infection or colonisation with multi-resistant organisms, and are more likely to be prescribed agents that are non-compliant with guidelines or are otherwise inappropriate compared with those with no penicillin allergy. ${ }^{10}{ }^{11}$ It is unclear if these inferior outcomes and increased use of antibiotics that are non-compliant to guidelines are the result of alternative agents being inherently less effective than penicillins at treating infection, or that clinicians are not selecting the most appropriate alternative agents for use in penicillin allergy as determined by experts in institutional or national guidelines. Data from point-prevalence surveys shows antibiotic treatment for respiratory tract infections $(28.9 \%)$, urinary tract infections (UTIs) $(13.5 \%)$ and skin and soft tissue infections (SSTIs) $(11 \%)$ account for half of all indications requiring antibiotic treatment in hospital. ${ }^{12} 13$ Trust guidelines recommend first-line antibiotics for these infections, usually penicillin or $\beta$-lactams, while for patient with penicillin allergy, alternative agents structurally unrelated to penicillin or $\beta$-lactams antibiotics are recommended. We sought to investigate the impact of penicillin allergy status on the selection of antibiotics received by hospital inpatients with concurrent infection with the frequently occurring conditions of SSTI, community-acquired pneumonia (CAP), hospital-acquired pneumonia (HAP) and UTI. The extent of detail recorded for patients with penicillin allergy at these trusts is unknown as are guideline-adherence rates for prescribing in penicillin allergy. Our hypothesis was that the selection of antibiotics by clinicians for patients with penicillin allergy would be less guideline adherent than those without penicillin allergy, and that there would be a high proportion of patient with inadequate description of their reported allergy.

\section{METHODS}

\section{Study objectives}

The objectives of this study were to (i) describe the documentation of allergy record in the electronic health record (EHR) and (ii) to determine to what extent clinicians treating patients with active infection in hospital are prescribing antibiotics adherent to local trust guidelines for those with (and without) penicillin allergy.

\section{Study design and dataset}

This was a cohort study that sourced patient data from two large tertiary referral National Health Service (NHS) hospital trusts in England. Data were extracted over two time-periods of 6-17 June 2016 and 13-24 February 2017. We studied all inpatients $\geq 18$ years with and without penicillin allergy with a current diagnosis of UTI, CAP, HAP and SSTI in the study period. Diagnoses were determined by treating clinicians. The indications were selected as these infections occur frequently and both trusts have clear guidance on alternate antibiotic for use in penicillin allergy for these indications. Both trusts had
EHRs in operation prior to and for the duration of the study period. Clinician access to guidelines was via trust intranet. Trust 1 data were sourced from point prevalence surveys where data were collected prospectively from ward visits Monday to Friday as previously described. ${ }^{14}$ Trust 2 data were captured retrospectively from an EHR report covering the full duration of each study period.

\section{Coding of allergy}

Documentation of allergy/adverse drug reaction (ADR) status was coded as 'allergy', 'side-effect', or 'no documentation', determined from information recorded in the allergy/ADR field and any corroborative information in the EHR.

\section{Assessment of antibiotics for adherence to trust guidelines}

While both trusts are NHS institutions, each had its own antibiotic guideline for treatment of infection. There were similarities in the antibiotic agents stated in both guidelines for the included indications of CAP, HAP, SSTI and UTI. However, some notable differences in recommended antibiotics between the two guidelines were present when treating, severe CAP in penicillin allergy; severe HAP when no allergy; complicated cellulitis in both penicillin allergy and no allergy; and for uncomplicated UTI (online supplementary material).

Medication orders were assessed for adherence of antibiotic agents to respective trust guidelines for those patients with reported penicillin allergy or for those without penicillin allergy, whichever applied by allergy status. If the antibiotic order was for the stated antibiotic in the respective trust's guideline, for the indication in question, it was assessed as adherent. Conversely, if a patient had not received the guideline recommended antibiotic, the antibiotic order was assessed as non-adherent. Adherence was assessed by experienced clinical pharmacists, who work closely with medical microbiologists and infectious diseases physicians in their clinical roles. Ambiguous cases were adjudicated by a second investigator.

\section{Patient and public involvement}

Patients and the public were not directly involved in this study; however, patient data resource and access were approved by the respective research governance offices of each institution.

\section{Statistical analysis}

Differences between groups in the frequency of patient characteristics were compared using a two-sample test of proportions. Differences between groups in age and other continuous variables were compared using an independent t-test. Adherence of antibiotics to trust guidelines was described using frequency counts and percentages. Differences in adherence between patients with and without penicillin allergy was assessed using univariate and multivariate (age and sex adjusted) binary logistic regression with results expressed as ORs with 95\% CIs. Robust standard errors were used to account 
All in-patients with documented: CAP, HAP, cellulitis or UTI receiving antibiotics

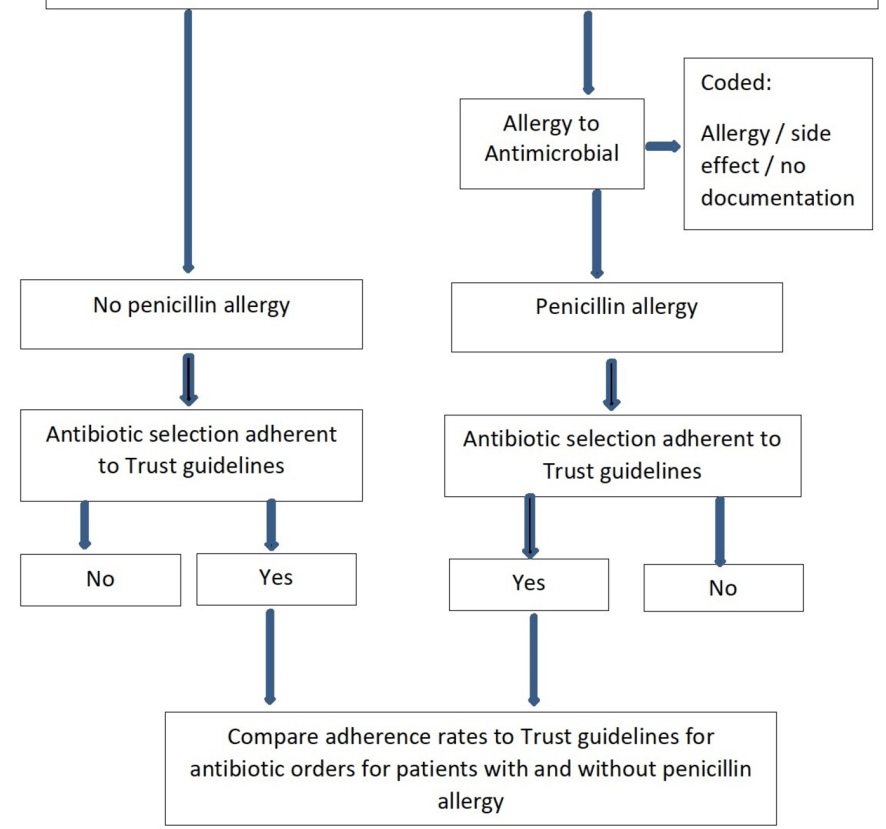

Figure 1 Study flow diagram. CAP, community acquired pneumonia; HAP, hospital acquired pneumonia; UTI, urinary tract infection.

for the clustering of prescriptions for a patient. Specialty of treating team was not available for all antibiotic order data, as such we were not able to include that variable in multivariate regression. A $p$ value $<0.05$ was used to define statistical significance. All statistical analyses were performed using STATA V.15.0 (StataCorp, Texas, USA).

\section{RESULTS}

\section{Patient characteristics}

There were 2645 antibiotic medication orders from a population of 1497 adult patients over the study duration. Study flow diagram is presented in figure 1 . Thirty-five paediatric patients were excluded. Overall, patients had a median age of 73 (IQR 56-82) years, $50.2 \%$ were female and $1131(75.5 \%)$ treated by medical teams. There were some differences observed for those with reported penicillin allergy; more female sex $60 \%$ versus $48 \% \mathrm{p}=0.001$, and notably more allergies to other antibiotics $34.6 \%$ versus $6.5 \% \mathrm{p} \leq 0.001$. Patients were treated for the indications of CAP $(495 ; 33.1 \%)$, UTI $(407 ; 27.2 \%)$, HAP $(330 ; 22 \%)$ and SSTI $(265 ; 17.7 \%)$. Patient characteristics overall and for those with and without reported penicillin allergy are presented in table 1.

\section{Allergy}

There were $341(22.7 \%)$ patients with a reported antimicrobial allergy. In the $240(16 \%)$ patients where penicillin allergy was reported in the EHR, the specific agent was recorded in $70(29.2 \%)$. The median age of patients with penicillin allergy was 73 years (58-85) and $60 \%$ female. Overall there were 175 non-penicillin antimicrobial allergies documented, with more allergies observed in those with reported penicillin; macrolides $(8.8 \%$ vs $1.4 \%$ $\mathrm{p} \leq 0.001)$; trimethoprim $(6.3 \%$ vs $1 \% \mathrm{p} \leq 0.001)$ and quinolones $(5.4 \%$ vs $1 \% \mathrm{p} \leq 0.001)$ accounting for half $(52 \%)$ of these allergies (table 1). Labelling of penicillin allergies was identified as allergy $(52 ; 21.7 \%)$, side effect $(27$; $11.3 \%$ ) and no documentation $(161 ; 67.1 \%)$ (table 2$)$. Frequency and per cent of reported ADR/allergy label for other antibiotics is presented for those with and without reported penicillin allergy (table 3 ).

\section{Assessment of antibiotics for adherence to trust guidelines}

Overall, 2184 of 2645 (82.6\%) of medication orders were adherent to trust antibiotic guidelines. Adherence of medication orders to trust antibiotic guidelines for all and by penicillin allergy status is shown in figure 2 . Overall adherence was greatest where penicillin allergy was recorded $(453$ of $517 ; 87.6 \%)$ versus no penicillin allergy (1731 of 2128; 81.3\%) (adjusted OR 0.52 (95\% CI 0.37 to 0.73$) \mathrm{p}<0.001)$. Adherence to guidelines for antibiotics prescribed for CAP was greater for those with penicillin allergy ( 151 of $163 ; 92.6 \%$ ) versus no penicillin allergy (582 of $810 ; 71.9 \%$ ) (adjusted OR 0.21 (95\% CI 0.11 to 0.37$) \mathrm{p}<0.001)$. Medication orders for UTI were more adherent to guidelines if patients had no penicillin allergy ( 486 of $539 ; 90.2 \%$ ) versus penicillin allergy (95 of $116 ; 81.9 \%$ ) (unadjusted OR 2.03 (95\% CI 1.11 to 3.60 ) $\mathrm{p}=0.011$ ), however, the there was no difference in the adjusted OR (OR $1.52(0.65-3.51) \mathrm{p}=0.332)$. For the indications of HAP and SSTI there was no differences between those with and without penicillin allergy (table 4).

\section{DISCUSSION}

In our study, almost one-quarter (23\%) of patients had an antimicrobial allergy label in the EHR and a staggering $16 \%$ had a penicillin allergy label. Our prevalence of penicillin allergy is considerable higher than the $10 \%$ figure often stated in the literature, ${ }^{15}$ yet very similar to a number of other hospital-based studies where penicillin allergy was found in $14 \%-16 \%$ of patients. ${ }^{16}{ }^{17}$ Furthermore, this incidence of penicillin allergy may reflect the relatively high median age of our population, as others have found increasing age to be associated with penicillin allergy. ${ }^{18}$ Penicillin allergy was overwhelmingly the most commonly reported antimicrobial allergy found, which is consistent with that reported by others. ${ }^{19}$ We were concerned to find that two-thirds of patients with a reported penicillin allergy had an absence of any description in the EHR regarding their allergy. Such a high proportion of patients with no further information on their allergy is alarming. Interestingly, others have also reported lack of allergy description regarding penicillin allergy in up to $80 \%$ of patients in hospital, ${ }^{20}$ and $77 \%$ of those in community care. ${ }^{9}$ While a record of penicillin allergy status may have been entered into the health record at a prior episode of care, and possibly a long time ago, ${ }^{21} 22$ it is incumbent on clinicians providing care 
Table 1 Patient characteristics, allergy status and indication for therapy

\begin{tabular}{|c|c|c|c|c|}
\hline Characteristic* $^{*}$ & All patients, $n=1497$ & $\begin{array}{l}\text { Reported penicillin } \\
\text { allergy, } n=240\end{array}$ & $\begin{array}{l}\text { No reported } \\
\text { penicillin } \\
\text { allergy, } n=1257\end{array}$ & $P$ value \\
\hline Age, years median (IQR) & 73 (56-82) & $73(55-84)$ & $73(55-84)$ & 0.745 \\
\hline Sex, female & $752(50.2)$ & $144(60)$ & $602(48)$ & 0.001 \\
\hline \multicolumn{5}{|l|}{ Specialty } \\
\hline Medicine & $683(45.6)$ & $109(45.4)$ & $574(45.6)$ & 0.944 \\
\hline Acute medicine & $295(19.7)$ & $49(20.4)$ & 246 (19.5) & 0.763 \\
\hline Elderly medicine & $153(10.2)$ & $23(9.6)$ & $130(10.3)$ & 0.029 \\
\hline Specialist surgical & $265(17.7)$ & $42(17.5)$ & $223(17.7)$ & $<0.001$ \\
\hline General surgical & $94(6.3)$ & $16(6.7)$ & $78(6.2)$ & 0.787 \\
\hline Private & $7(0.5)$ & $1(0.4)$ & $6(0.5)$ & 1.000 \\
\hline $\begin{array}{l}\text { Antibiotic allergies/adverse drug } \\
\text { reaction }\end{array}$ & $165(11)$ & $83(34.6)$ & $82(6.5)$ & $<0.001$ \\
\hline Macrolides & $39(2.6)$ & $21(8.8)$ & $18(1.4)$ & $<0.001$ \\
\hline Trimethoprim & $27(1.8)$ & $15(6.3)$ & $12(1)$ & $<0.001$ \\
\hline Quinolones & $25(1.7)$ & $13(5.4)$ & $12(1)$ & $<0.001$ \\
\hline Nitrofurantoin & $18(1.2)$ & $9(3.8)$ & $9(0.7)$ & $<0.001$ \\
\hline Sulfonamides & $17(1.1)$ & $6(2.5)$ & $11(0.9)$ & 0.029 \\
\hline Tetracyclines & $7(0.5)$ & $4(1.7)$ & $3(0.2)$ & 0.015 \\
\hline Glycopeptide & $7(0.5)$ & $1(0.4)$ & $6(0.5)$ & 0.688 \\
\hline Cephalosporins & $7(0.5)$ & $5(2.1)$ & $2(0.2)$ & 0.002 \\
\hline Metronidazole & $6(0.4)$ & $3(1.3)$ & $3(0.2)$ & 0.056 \\
\hline Meropenem & $5(0.3)$ & $4(1.7)$ & $1(0.1$ & 0.003 \\
\hline Clindamycin & $4(0.3)$ & $1(0.4)$ & $3(0.2)$ & 0.503 \\
\hline Aminoglycoside & $3(0.2)$ & $1(0.4)$ & $2(0.2)$ & 0.408 \\
\hline \multicolumn{5}{|l|}{ Indications for antibiotic treatment } \\
\hline Community-acquired pneumonia & $495(33.1)$ & $73(30.4)$ & $422(33.6)$ & 0.341 \\
\hline Urinary tract infection & $407(27.2)$ & $64(26.7)$ & $343(27.3)$ & 0.843 \\
\hline Hospital-acquired pneumonia & $330(22)$ & $55(22.9)$ & 275 (21.9) & 0.143 \\
\hline $\begin{array}{l}\text { Bacterial skin and soft tissue } \\
\text { infection }\end{array}$ & $265(17.7)$ & $50(20.8)$ & $215(17.1)$ & 0.165 \\
\hline
\end{tabular}

*Data reported as $\mathrm{n}(\%)$ unless otherwise stated.

tIndependent samples t-test or a two-sample test of proportions as appropriate.

do more to enquire, contact the provider facility where the event was first recorded, and detail the nature of the penicillin reaction. At a very minimum, clinicians should describe what steps were taken to clarify the record, and if the patient is suitable for referral to allergy or immunology to confirm or de-label their reported penicillin allergy. ${ }^{16}$ This approach has increasingly been advocated as an antimicrobial stewardship strategy, ${ }^{23}$ and has been successfully adopted by others. ${ }^{24}$

In this study, we found that patients with penicillin allergy overall received antibiotics that were significantly more adherent to guidelines $(88 \%)$ when compared with those without penicillin allergy. This finding was higher than that reported in a small hospital study $(n=94)$, investigating clinical implications of $\beta$-lactam allergy, which found adherence to antibiotic guidelines was $81 \%$, however, patients without penicillin allergy were not included in that study. ${ }^{25}$

We hypothesised that patients with penicillin allergy would be prescribed antibiotics that were overall less guideline adherent than those with no penicillin allergy. Our hypothesis was based on a recent large study $(n=21031)$ that found patients with documented antimicrobial allergy had less guideline compliant agents prescribed compared with those without allergy $(78.7 \%$ vs $79.2 \%, p=0.001) .{ }^{10}$ Our hypothesis was initially observed for patients with UTI, where penicillin allergy was associated with receiving less guideline adherent antibiotics, however, when we performed multivariate analysis, the adjusted ORs did not show any effect. 
Table 2 Reported reaction to penicillin and identified agents

\begin{tabular}{lc}
\hline Reported reaction to penicillin & $\mathbf{n = 2 4 0}$ \\
\hline Allergy & $52(21.7)$ \\
Side effect & $27(11.3)$ \\
No documentation & $161(67.1)$ \\
\hline Penicillin agent reported as allergic & \\
\hline Penicillin (unspecified) & $170(70.8)$ \\
Co-amoxiclav & $20(8.3)$ \\
\hline Piperacillin-tazobactam & $18(7.5)$ \\
Amoxicillin/ampicillin & $17(7.1)$ \\
\hline Flucloxacillin & $14(5.8)$ \\
\hline Phenoxymethylpenicillin & $1(0.4)$ \\
\hline
\end{tabular}

Our finding that patients with penicillin allergy overall received antibiotics that were more guideline-adherent was driven by patients being treated for CAP. While patients with reported penicillin allergy received antibiotics that were more guideline adherent for CAP, those patients were receiving macrolides, tetracyclines and or quinolones over first-line amoxicillin-based therapy. Additionally, patients with penicillin allergy received clindamycin or a macrolide rather than first-line flucloxacillin in cellulitis, and nitrofurantoin or quinolones for UTI rather than penicillin-based agent. The high level of prescribing adherence to treatment guidelines in CAP for those with penicillin allergy, may have been due to trust guidelines typically recommending a single antibiotic, except for those with the most severe presentations of CAP. This is in contrast to guideline recommendations for those without allergy, where guidelines suggest two antibiotics for treatment of CAP for all but the least severe presentations. Thus, it may have been easier for clinicians to prescribe one antibiotic in penicillin allergy, rather than two for those without allergy. Furthermore, it is also possible that clinicians were less certain of which antibiotic to prescribe in penicillin allergy and therefore consulted trust guidelines more readily than they did for those without allergy.

When interpreting our findings, both participating trusts had locally endorsed guidelines, which were readily

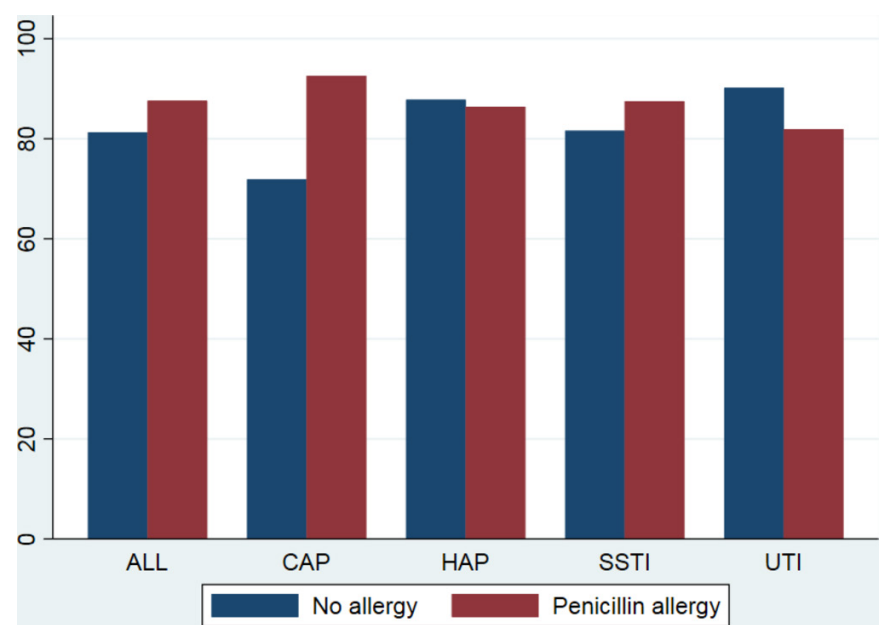

Figure 2 Adherence of medication orders to trust antibiotic guidelines by penicillin allergy status. CAP, community acquired pneumonia; HAP, hospital acquired pneumonia; SSTI, skin and soft tissue infection; UTI, urinary tract infection.

accessible, containing clear advice on alternative agents to use for patients with penicillin allergy for all the indications included in this study. Studies describing poor outcomes and greater use of non-compliant antibiotics in hospitalised, in often complex, immunocompromised patients frequently provide little or no detail about the presence or access to endorsed guidelines, or guideline adherence in those respective institutions, or if such guidelines provide advice on penicillin allergy for the specific indications in question. ${ }^{10} 1626-28$

While our study was conducted in tertiary institutions, we deliberately selected frequently occurring indications that are managed in large and small facilities across the county. We propose freely accessible, updated and endorsed antibiotic guidelines with clear alternative recommendations in penicillin allergy for most indications, would be a valuable recommendation for hospitals that do not currently have these available. Furthermore, we recommend better allergy documentation, and testing. Providing ready-access to other contemporaneous guidelines may go some way to limiting the inferior outcomes observed for those with penicillin allergy. Vancomycin has been reported as the most frequently used non $\beta$-lactam

Table 3 Frequency and per cent of documented adverse drug reaction/allergy labels for non-penicillin antibiotics

\begin{tabular}{|c|c|c|c|c|}
\hline & All patients & $\begin{array}{l}\text { Reported penicillin } \\
\text { allergy }\end{array}$ & $\begin{array}{l}\text { No reported } \\
\text { penicillin allergy }\end{array}$ & P value* \\
\hline Non-penicillin antibiotic allergy† & 175 & 83 & 92 & \\
\hline Side effect & $28(16)$ & $9(10.8)$ & $19(20.7)$ & 0.077 \\
\hline No documentation & $125(71.4)$ & $62(74.7)$ & $63(68.5)$ & 0.363 \\
\hline
\end{tabular}

Data reported as $\mathrm{n}(\%)$.

${ }^{*}$ Two-sample test of proportions.

†Ten patients have both allergy and side effect reported. 
Table 4 OR of receiving antibiotics adherent to trust guidelines for all antibiotic orders and by reported penicillin allergy status

\begin{tabular}{|c|c|c|c|c|c|c|c|}
\hline $\begin{array}{l}\text { Indication for } \\
\text { antibiotic }\end{array}$ & $\begin{array}{l}\text { All } \\
\text { antibiotics }\end{array}$ & $\begin{array}{l}\text { No reported } \\
\text { penicillin } \\
\text { allergy }\end{array}$ & $\begin{array}{l}\text { Reported } \\
\text { penicillin } \\
\text { allergy }\end{array}$ & $\begin{array}{l}\text { Unadjusted } \\
\text { OR }(95 \% \mathrm{CI})^{*}\end{array}$ & P value* & $\begin{array}{l}\text { Adjusted OR } \\
(95 \% \mathrm{Cl})\end{array}$ & $\mathrm{P}$ value \\
\hline $\begin{array}{l}\text { Antibiotic orders } \\
\text { assessed }(n=2645)\end{array}$ & 2184 (82.6) & $\begin{array}{l}1731 / 2128 \\
(81.3)\end{array}$ & $453 / 517$ (87.6) & 0.61 (0.46 to 0.82 ) & $<0.001$ & 0.52 (0.37 to 0.73$)$ & $<0.001$ \\
\hline $\begin{array}{l}\text { Skin and soft tissue } \\
\text { infection }(n=457)\end{array}$ & 380 (83.2) & 275/337 (81.6) & $105 / 120(87.5)$ & 0.63 (0.32 to 1.19$)$ & 0.138 & 0.61 (0.32 to 1.18$)$ & 0.142 \\
\hline $\begin{array}{l}\text { Hospital-acquired } \\
\text { pneumonia }(n=560)\end{array}$ & $490(87.5)$ & $388 / 442(87.8)$ & 102/118 (86.4) & 1.13 (0.58 to 2.10$)$ & 0.70 & 1.19 (0.65 to 2.19$)$ & 0.570 \\
\hline $\begin{array}{l}\text { Urinary tract infection } \\
(\mathrm{n}=655)\end{array}$ & $581(88.7)$ & $486 / 539$ (90.2) & 95/116 (81.9) & 2.03 (1.11 to 3.60$)$ & 0.011 & 1.52 (0.65 to 3.51$)$ & 0.332 \\
\hline
\end{tabular}

Data reported as $\mathrm{n}(\%)$.

*Estimates from logistic regression model with robust standard errors.

†Estimates from logistic regression model with adjustment for age and sex and with robust standard errors.

OR, odds ratio.

agent in hospitalised patients with penicillin allergy treated for infection, ${ }^{6} 27$ with rates of usage in those with penicillin allergy ranging from $39 \%-$ to $66 \% .{ }^{1726}$ Vancomycin is known to be an inferior agent in the prevention of surgical site infections by meticillin-sensitive Staphylococcus aureus compared with penicillins and $\beta$-lactams. ${ }^{29}$ Additionally, a recent study found patients with reported penicillin allergy undergoing surgery had an increased risk of surgical site infection compared with those with no allergy when prescribed antibiotic prophylaxis with alternate agents such vancomycin. ${ }^{30}$

The current study has a number of strengths. Data were collected from patients treated at multiple sites, in different organisations from in two geographical regions of England. Furthermore, this is meaningful as most of the data on this topic is from outside England. While the study was conducted in tertiary referral centres, the infections treated are common and likely to be treated in all hospitals regardless of size, which gives strength to the generalisability of the results.

\section{Limitations}

This work is not without limitations. The study included retrospective data and was a cohort study, which cannot exclude confounding bias. No information on comorbidities, presence of febrile illness, immune suppression, or microbiology was collected which may have influenced the selection of antibiotics and ultimately guideline adherence rates. During multivariate regression model, we were unable to include the specialty of treating teams in the model as this data variable was not available for all antibiotic orders. Following-up clinical data and patient outcomes were not assessed in this study; further studies in this field should consider including outcomes such as length of stay, readmission rates, treatment failures and ADRs.

\section{CONCLUSION}

In this study, we found a high proportion of hospital inpatients had reported penicillin compared with the known prevalence of penicillin allergy in the community. Importantly two-thirds of these penicillin allergies had no further details whatsoever of the nature of the allergy in the patients' EHR. This lack of information leads to uncertainty in making clinical decisions and may have important implications for patient safety. In contrast to previous studies, in the current study we found that patients with a reported penicillin allergy were more likely to be prescribed antibiotics that were adherent to local guidelines, which may reflect the existence of unambiguous detailed guidance for antibiotic selection in penicillin allergy in the study institutions. Future studies on prescribing in penicillin allergy should include data on adherence to guidelines and seek to further understand the implications of this effect.

\section{Author affiliations}

${ }^{1}$ Imperial College Healthcare NHS Trust, London, UK

${ }^{2} \mathrm{NIHR}$, Health Protection Research Unit in Healthcare Associated Infections and

Antimicrobial Resistance, Imperial College London, London, UK

${ }^{3}$ Clinical Microbiology and Public Health Laboratory, National Infection Service,

Public Health England, Cambridge University Hospitals NHS Foundation Trust,

Cambridge, UK

${ }^{4}$ Department of Medicine, University of Cambridge, Cambridge, UK

${ }^{5}$ College of Medicine and Public Health, Flinders University, Adelaide, South Australia, Australia

${ }^{6}$ Centre for Medication Safety and Service Quality, Imperial College Healthcare NHS Trust, London, UK

${ }^{7}$ Research Department of Practice and Policy, UCL School of Pharmacy, London, UK ${ }^{8}$ Pharmacy Department, Cambridge University Hospitals NHS Foundation Trust, Cambridge, UK

Acknowledgements CJP, BDF, MG and AHH are affiliated to the National Institute for Health Research (NIHR) Health Protection Research Unit (HPRU) in Healthcare Associated Infection and Antimicrobial Resistance at Imperial College London in partnership with Public Health England (PHE) and the NIHR Imperial Patient Safety Translational Research Centre. AHH is a NIHR Senior Investigator. The 
authors kindly acknowledge Ms Orla Geoghegan, Pharmacy Department, Imperial College Healthcare NHS Trust, London, UK for contribution with data collection and Professor Richard Woodman, Flinders Centre for Epidemiology and Biostatistics, Flinders University, Adelaide, Australia for his statistical advice. Preliminary findings of this work were presented at the Federation of Infection Societies Conference in Birmingham, UK, 30 November to 2 December 2017 (abstract 184).

Contributors CJP, FJC, BDF, ETB and AHH conceived the study. CJP, MG, FJC, BDF, DAE, MEM, RS, ETB and AHH contributed to the design of the study. CJP, MG, RS and MEM were involved with data acquisition. CJP performed data analysis, and CJP, FJC, DAE, BDF and AHH interpreted the data. CJP wrote the initial manuscript draft and all authors contributed to critical revision of the manuscript for important intellectual content. RS provided administrative support. All authors approved the final version of the manuscript.

Funding This article presents research funded in part by an Endeavour Executive Fellowship (number 6040) from the Australian Government. The funders had no role in study design, data collection and analysis, decision to publish, or preparation of the manuscript.

Disclaimer The views expressed are those of the authors and not necessarily those of the NHS, the NIHR, the Department of Health and Care or Public Health England.

Competing interests None declared.

Patient consent for publication Not required.

Ethics approval The study was approved by both institutions' Clinical Audit/ Research Governance offices (reference numbers Trust 1 HSI004/ Trust 2 PRN6465).

Provenance and peer review Not commissioned; externally peer reviewed.

Data sharing statement Please contact the corresponding author to discuss requests for availability of datasets analysed during this study. Request consideration will be in accordance with English National Health Service (NHS) research governance criteria.

Open access This is an open access article distributed in accordance with the Creative Commons Attribution Non Commercial (CC BY-NC 4.0) license, which permits others to distribute, remix, adapt, build upon this work non-commercially, and license their derivative works on different terms, provided the original work is properly cited, appropriate credit is given, any changes made indicated, and the use is non-commercial. See: http://creativecommons.org/licenses/by-nc/4.0/.

\section{REFERENCES}

1. Drug and Therapeutics Bulletin. Penicillin allergy-getting the label right. BMJ 2017;358:j3402.

2. National Institute for Health and Clinical Excellence. Drug allergy: diagnosis and management of drug allergy (CG183). 2014 https:// www.nice.org.uk/guidance/cg183.

3. Kerr JR. Penicillin allergy: a study of incidence as reported by patients. Br J Clin Pract 1994;48:5-7.

4. Yates $A B$. Management of patients with a history of allergy to betalactam antibiotics. Am J Med 2008;121:572-6.

5. Koliscak LP, Johnson JW, Beardsley JR, et al. Optimizing empiric antibiotic therapy in patients with severe $\beta$-lactam allergy. Antimicrob Agents Chemother 2013;57:5918-23.

6. Macy E, Contreras R. Health care use and serious infection prevalence associated with penicillin "allergy" in hospitalized patients: A cohort study. J Allergy Clin Immunol 2014;133:790-6.

7. Macy $\mathrm{E}$. The clinical evaluation of penicillin allergy: what is necessary, sufficient and safe given the materials currently available? Clin Exp Allergy 2011;41:1498-501.
8. Mirakian R, Leech SC, Krishna MT, et al. Management of allergy to penicillins and other beta-lactams. Clin Exp Allergy 2015;45:300-27.

9. Shah NS, Ridgway JP, Pettit N, et al. Documenting penicillin allergy: the impact of inconsistency. PLoS One 2016;11:e0150514.

10. Trubiano JA, Chen C, Cheng AC, et al. Antimicrobial allergy 'labels' drive inappropriate antimicrobial prescribing: lessons for stewardship. J Antimicrob Chemother 2016;71:1715-22.

11. Charneski L, Deshpande G, Smith SW. Impact of an antimicrobia allergy label in the medical record on clinical outcomes in hospitalized patients. Pharmacotherapy 2011;31:742-7.

12. Ansari F, Erntell M, Goossens $H$, et al. The European surveillance of antimicrobial consumption (ESAC) point-prevalence survey of antibacterial use in 20 European hospitals in 2006. Clin Infect Dis 2009;49:1496-504

13. Oza A, Hogan-Murphy D. National Annual Antimicrobial Point Prevalence Survey 2016. Dublin, Ireland: Disease Survelliance Report of the Health Protection Survelliance Centre, 2016.

14. Dean B, Lawson W, Jacklin A, et al. The use of serial pointprevalence studies to investigate hospital anti-infective prescribing. Int J Pharm Pract 2002;10:121-5.

15. Solensky R. Hypersensitivity reactions to beta-lactam antibiotics. Clin Rev Allergy Immunol 2003;24:201-20.

16. Conway EL, Lin K, Sellick JA, et al. Impact of penicillin allergy on time to first dose of antimicrobial therapy and clinical outcomes. Clin Ther 2017;39:2276-83.

17. Lee CE, Zembower TR, Fotis MA, et al. The incidence of antimicrobial allergies in hospitalized patients: implications regarding prescribing patterns and emerging bacterial resistance. Arch Intern Med 2000;160:2819-22.

18. Macy E, Poon K-Y T. Self-reported antibiotic allergy incidence and prevalence: age and sex effects. Am J Med 2009;122:778.e1-7.

19. Pongdee T, Li JT. Evaluation and management of penicillin allergy. Mayo Clin Proc 2018;93:101-7.

20. Sade K, Holtzer I, Levo Y, et al. The economic burden of antibiotic treatment of penicillin-allergic patients in internal medicine wards of a general tertiary care hospital. Clin Exp Allergy 2003;33:501-6.

21. Trubiano JA, Adkinson NF, Phillips EJ. Penicillin allergy is not necessarily forever. JAMA 2017;318:82-3.

22. Borch JE, Andersen KE, Bindslev-Jensen C. The prevalence of suspected and challenge-verified penicillin allergy in a university hospital population. Basic Clin Pharmacol Toxicol 2006;98:357-62.

23. Trubiano J, Phillips E. Antimicrobial stewardship's new weapon? A review of antibiotic allergy and pathways to 'de-labeling'. Curr Opin Infect Dis 2013;26:526-37.

24. Blumenthal KG, Shenoy ES, Varughese CA, et al. Impact of a clinical guideline for prescribing antibiotics to inpatients reporting penicillin or cephalosporin allergy. Ann Allergy Asthma Immunol 2015;115:294-300. e292.

25. Satta $G$, Hill V, Lanzman $M$, et al. $\beta$-lactam allergy: clinical implications and costs. Clin Mol Allergy 2013;11:2

26. Huang KG, Cluzet V, Hamilton K, et al. The Impact of Reported Beta-Lactam Allergy in Hospitalized Patients With Hematologic Malignancies Requiring Antibiotics. Clin Infect Dis 2018;67:27-33.

27. Picard M, Bégin $\mathrm{P}$, Bouchard $\mathrm{H}$, et al. Treatment of patients with a history of penicillin allergy in a large tertiary-care academic hospital. $J$ Allergy Clin Immunol Pract 2013;1:252-7.

28. Trubiano JA, Leung VK, Chu MY, et al. The impact of antimicrobial allergy labels on antimicrobial usage in cancer patients. Antimicrob Resist Infect Control 2015;4:23.

29. Bull AL, Worth LJ, Richards MJ. Impact of vancomycin surgical antibiotic prophylaxis on the development of methicillinsensitive staphylococcus aureus surgical site infections: report from Australian Surveillance Data (VICNISS). Ann Surg 2012;256:1089-92.

30. Blumenthal KG, Ryan EE, Li Y, et al. The impact of a reported penicillin allergy on surgical site infection risk. Clin Infect Dis 2018;66:329-36. 